\title{
Negatively buoyant starting jets
}

\author{
C. Marugán-Cruz, ${ }^{1, a)}$ J. Rodríguez-Rodríguez, ${ }^{1}$ and C. Martínez-Bazán ${ }^{2}$ \\ ${ }^{1}$ Grupo de Mecánica de Fluidos, Universidad Carlos III de Madrid, 28911 Leganés, Spain \\ ${ }^{2}$ Departamento de Ingeniería Mecánica y Minera, Área de Mecánica de Fluidos, Universidad de Jaén, \\ Campus de las Lagunillas, 23071 Jaén, Spain
}

(Received 4 February 2009; accepted 23 September 2009; published online 2 November 2009)

\begin{abstract}
The initial development of negatively buoyant jets has been investigated experimentally and numerically, focusing on the role played by gravity in the evolution of the leading vortex ring. Under the experimental conditions considered in this work, the densimetric Froude number, Fr $=\rho_{j} U_{j}^{2} /\left[\left(\rho_{0}-\rho_{j}\right) g D\right]$, which represents the ratio between the jet momentum and the buoyancy forces, emerges as the most relevant parameter characterizing the dynamics of the flow. Two different flow regimes have been observed depending on the Froude number: for sufficiently small Fr, the vortex ring generated initially is pushed radially away by gravity forces before it has time to detach from the shear layer originating at the orifice. On the other hand, when the Froude number is larger than a critical value, $\mathrm{Fr}>\mathrm{Fr}_{c} \sim 1$, the vortex ring detaches from the injection orifice and propagates downstream into the stagnant ambient followed by a trailing jet until it eventually reaches a maximum penetration depth. In order to clarify the mechanisms leading to the transition between the two regimes, and to gain physical understanding of the formation dynamics of negatively buoyant starting jets, the total and the vortex circulation, as well as the trajectory of the vortex center, have been measured and compared to the case of neutrally buoyant jets. Finally, based on the experimental measurements and on the results of the numerical computations, a kinematic model that successfully describes the evolution of both total circulation and vortex trajectory is proposed. () 2009 American Institute of Physics. [doi:10.1063/1.3253690]
\end{abstract}

\section{INTRODUCTION}

The transient discharge of a jet of the same density and temperature as the ambient has been the subject of many investigations. ${ }^{1,2}$ The interest on transient jets arises from the fact that these flows are found in many industrial applications such as the injection of reactants in combustion chambers or in nature, for instance, as the propulsion mechanism of marine species. Moreover, the study of starting jets is deeply related with the formation of vortex rings. ${ }^{3}$ In fact, a typical experimental configuration to generate vortex rings is that of a piston moving inside a cylinder that pushes a certain volume of fluid into a stagnant pool containing the same fluid. ${ }^{4}$ Near the injection orifice, the boundary layer rolls up into a vortex ring and, if the piston stroke is long enough, this vortex is followed by a trailing jet that keeps injecting vorticity into the leading vortex. At some point, the leading vortex ring has engulfed enough vorticity to develop a selfinduced velocity faster than the injection velocity, which eventually will lead to its detachment from the trailing jet (pinch-off). ${ }^{1}$ The value of the dimensionless time at which the vortex ring stops entraining vorticity is commonly known as formation number, ${ }^{1} F$, and it is an important characteristic of starting jets, as it determines the maximum size of vortex ring that can be achieved under a given set of conditions. The value of $F$ is typically 4 , however, the time evolution of the piston velocity or the velocity profile at the injection orifice can alter this value. ${ }^{1,5}$ Moreover, Zhao et al. ${ }^{5}$ and Pawlak

\footnotetext{
${ }^{a)}$ Electronic mail: cmarugan@ing.uc3m.es. URL: http://fluidos.uc3m.es/ cmarugan.html.
}

et $a l .{ }^{6}$ indicated that secondary instabilities caused by perturbations in the flow could also lead to variations in the formation number of approximately $20 \%$.

When the jet density differs from that of the ambient, buoyancy forces must be taken into account. Positively buoyant jets are generated when the fluid injected upward (downward) is lighter (heavier) than the ambient fluid ${ }^{7}$ and, therefore, the inertia and buoyancy forces act in the same direction. However, if the buoyancy forces oppose to the inertia forces, one finds negatively buoyant jets or fountains. Plumes and other environmental flows such as volcanic eruptions or certain behavior of clouds are examples where buoyancy affects, by opposing or contributing, to the motion of the fluids. ${ }^{8}$

Most of the studies of negatively buoyant jets have been focused on the parameters controlling the penetration height. To measure the maximum penetration distance of turbulent fountains, Turner ${ }^{8}$ performed a series of experiments injecting upward a salt water jet into a tank containing fresh water. He found that turbulent fountains were independent of the Reynolds number, $\operatorname{Re}=\rho_{j} U_{j} D / \mu_{j}$, where $\rho_{j}$ and $U_{j}$ are the density and velocity of the jet at the injection, respectively, $\mu_{j}$ is the jet viscosity, and $D$ is the diameter of the discharging orifice, and that their general behavior only depended on the densimetric Froude number, $\mathrm{Fr}=\rho_{j} U_{j}^{2} /\left[\left(\rho_{0}-\rho_{j}\right) g D\right]$, where $\left(\rho_{0}-\rho_{j}\right)$ is the density difference between the ambient and the jet and $g$ is the gravitational acceleration. In particular, he determined that the penetration height depended on the Froude number as $x_{s t} / D \propto \mathrm{Fr}^{1 / 2}$ for large Froude numbers. Note that in the present work we have defined the Froude 
number as $\mathrm{Fr}=\rho_{j} U_{j}^{2} /\left[\left(\rho_{0}-\rho_{j}\right) g D\right]$, while other authors commonly presented their results in terms of the Richardson number $\mathrm{Ri}=\left(\rho_{0} / \rho_{j}-1\right) g D / U_{j}^{2}$, which relates to our Froude number as $\mathrm{Ri}=\mathrm{Fr}^{-1}$, or in terms of a Froude number given by $\mathrm{Fr}=U_{j} /\left(g^{*} D\right)^{1 / 2}$ with $g^{*}=\left(\rho_{0} / \rho_{j}-1\right) g$. Thus, to compare with our results, we will express their Richardson or Froude numbers in terms of the Froude number used here. Pantzlaff and Lueptow $^{7}$ studied the transient regime of positively and negatively buoyant turbulent jets, with Reynolds numbers varying from 2500 to 21000 . At their lower range of Reynolds numbers, and for negatively buoyant jets corresponding to $125<\mathrm{Fr}<3040$, they characterized the time evolution of the penetration distance of the jet before reaching the stationary height, around which the jet began to oscillate. Philippe et al. ${ }^{9}$ studied negatively buoyant jets at low Reynolds numbers $(\operatorname{Re}<350)$, for which the jet remains slender everywhere except at its head. They proposed a model for the initial phase of penetration, the final height, and the final jet profile and reported a dependence of the final jet penetration with Re at very low Reynolds numbers. In the numerical simulations by Lin and Armfield, ${ }^{10}$ where the Reynolds number varied in the range $200<\operatorname{Re}<800$, they also acknowledged the existence of a maximum penetration height before reaching the stationary value. However, in their work the dependence of the final height of the fountain with the Reynolds number was found to be very weak.

Cresswell and Szczepura ${ }^{11}$ measured the mean and fluctuating velocity, and temperature profiles using laser Doppler anemometry and thermocouples at the steady state of a negatively buoyant turbulent jet of $\operatorname{Re}=5000$ and $\mathrm{Fr} \sim 4.9$, and showed that there was no similarity profile in any of the regions of the flow and that axial gradients were important and could not be neglected.

Iglesias et al. ${ }^{12}$ studied numerically starting gas jets whose temperature was different from that of the ambient, and proposed a model for the total circulation of the jet in absence of gravity. Satti and Agrawal ${ }^{13}$ were also concerned with the variations of vorticity fields due to buoyancy effects in laminar starting jets, although their computational study was focused only on positively buoyant jets.

To our knowledge, most of the studies reported in literature aiming at the understanding of the buoyancy effects have been focused on fully turbulent jets and less interest has been paid to negatively buoyant jets at moderate Reynolds numbers. However, in the cases studied here, the Reynolds numbers were not sufficiently large, $800<\operatorname{Re}<2500$, to have a fully developed turbulent starting jet and, consequently, the flow could be considered laminar.

In order to identify the dimensionless parameters affecting the dynamics of starting buoyant jets, dimensional analysis can be applied to a characteristic magnitude, i.e., the steady penetration depth, $x_{\mathrm{st}}$. Thus, $x_{\mathrm{st}}$ will depend on the jet density, $\rho_{j}$, the ambient density, $\rho_{0}$, the jet viscosity, $\mu_{j}$, the ambient viscosity, $\mu_{0}$, the mean injection velocity, $U_{j}$, the diameter of the injection orifice, $D$, and the gravitational acceleration, $g$,

$$
x_{\mathrm{st}}=f_{1}\left(\rho_{j}, \rho_{0}, \mu_{j}, \mu_{0}, D, g, U_{j}\right) .
$$

Forming dimensionless groups the equation above yields

$$
x_{\mathrm{st}}^{*}=\frac{x_{\mathrm{st}}}{D}=f_{2}\left(\operatorname{Re}, \operatorname{Fr}, \frac{\mu_{0}}{\mu_{\mathrm{j}}}, \frac{\rho_{0}}{\rho_{\mathrm{j}}}\right) .
$$

In the experimental setup employed in this work the ambient-to-jet density ratio, $\rho_{0} / \rho_{j}$, could not be modified independently from the Reynolds and Froude numbers. However, the effect of $\rho_{0} / \rho_{j}$ in the flow conditions explored is expected to be negligible. Indeed, the density ratio was never varied more than $30 \%$, which, according to Iglesias et al. ${ }^{12}$ is small to induce significant differences in the flow, of the order of those obtained in this work varying the Froude number.

To evaluate the effect of the Reynolds number, several experiments and simulations were performed varying the Reynolds number while keeping constant the Froude number. In agreement with the results of previous investigations in homogeneous jets, the effect of the Reynolds number is negligible at large Re. For the same reason, the ambient-tojet viscosity ratio, $\mu_{0} / \mu_{j}$, is not expected to have an important role in the formation of negatively buoyant jets under the conditions explored in this work. Finally, as the difference of density of the fluids is caused by the different composition, the thermal diffusion has been also neglected.

In our study, Sec. II describes the experimental setup and the experimental techniques used to perform the measurements. Section III includes experimental visualizations of the time evolution of the jet for different Froude numbers. A description of the numerical method used is detailed in Sec. IV and the most important results and conclusions are summarized and discussed in Secs. V-VII.

\section{EXPERIMENTAL SETUP}

To generate jets under well defined conditions, a computer controlled piston moving inside a vertical cylinder was used to inject downward different ethanol/water solutions into an acrylic tank filled with fresh water (ambient fluid). The fluid inside the cylinder discharged into the tank through a circular orifice placed at the center of the upper wall, whose diameter could be varied among fixed values, i.e., $D=2,1.5$, and $1 \mathrm{~cm}$ (see Fig. 1). To avoid any effect of the tank walls on the dynamics of the jet, its dimensions, $60 \times 60 \times 100 \mathrm{~cm}^{3}$, were much larger than the characteristic diameter of the jet. The tank was hermetic except for the orifice and four windows located near the bottom, built with the aim of evacuating the ambient fluid at a rate equal to the injected volumetric flow rate. These windows connected with an open water pool where the tank was fully submerged.

Besides the orifice diameter and the jet injection velocity, the jet density was also varied in the experiments to obtain the desired range of nondimensional parameters characteristic of buoyant jets. To do so, the cylinder was filled with ethanol/water solutions with alcohol concentrations varying from $4 \%$ to $100 \%$ in weight, thus allowing dimensionless density differences in the range $10^{-4}<\Delta \rho / \rho_{j}<3$ $\times 10^{-1}$, with $\Delta \rho=\rho_{0}-\rho_{j}$. A guillotine, flush to the wall, was necessary to fill the feeding cylinder with the desired solution, but once the cylinder was filled, the guillotine was open and the water inside the tank was left to become quiescent. 


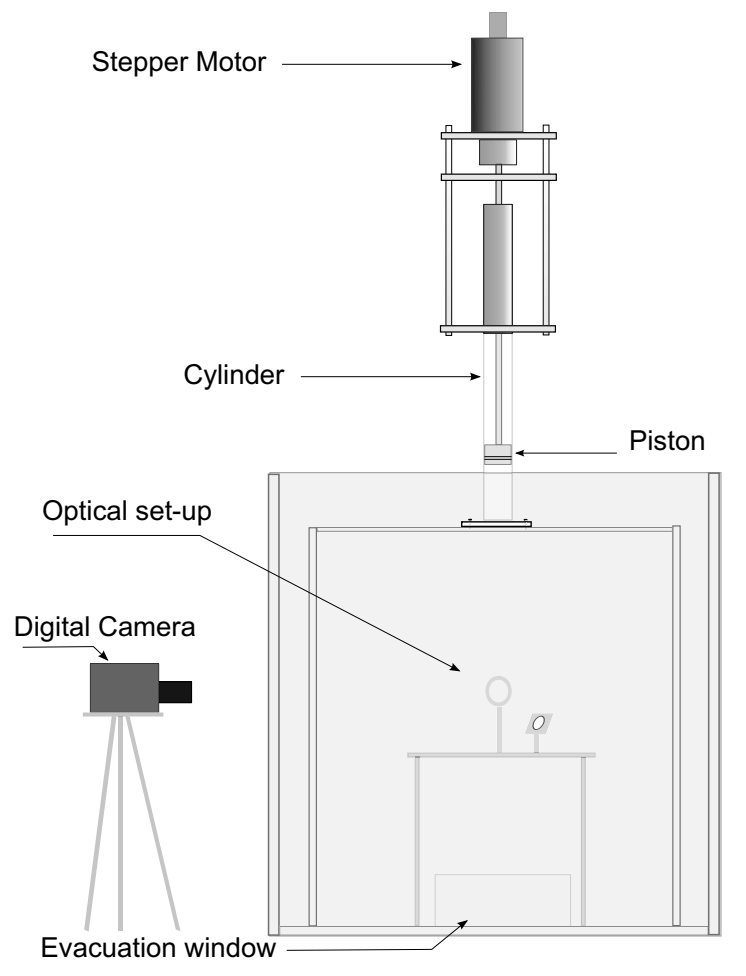

FIG. 1. Sketch of the experimental facility.

Properly choosing the jet injection velocity, $U_{j}$, the orifice diameter, $D$, and the jet density, $\rho_{j}$, the jet Reynolds number could be varied from 800 to 2500 , whereas the densimetric Froude number ranged from $0.2<\mathrm{Fr}$ to $\mathrm{Fr}=\infty$, this latter value corresponding to jets of the same density as that of the ambient fluid, $\rho_{j}=\rho_{0}$. Notice that $\mathrm{Fr}=\infty$, which corresponds to a Richardson number equal to zero, is not necessarily associated to a high Reynolds number and represents a homogeneous jet, i.e., a jet that discharges into a stagnant pool of the same liquid. Table I shows the values of the physical and dimensionless parameters of the experiments presented in this work. To check the repeatability of the experimental results, each session of experiments was performed several times under the same conditions.

Due to the initial acceleration of the piston the stationary exit velocity was not instantaneously reached but it was achieved after a short period of time, $t_{1}$. The mean exit velocity can therefore be written as

$$
u(t)=\left\{\begin{array}{cc}
U_{j} t / t_{1}, & t \leq t_{1} \\
U_{j}, & t>t_{1},
\end{array}\right.
$$

where $t_{1}$ is the acceleration time. Thus, to take into account this initial acceleration phase, the time was made dimensionless using the following expression:

$$
\hat{t}=\left\{\begin{array}{cc}
t^{2} / 2 t_{1} U_{j} / D, & t \leq t_{1} \\
\left(t-t_{1} / 2\right) U_{j} / D, & t>t_{1},
\end{array}\right.
$$

which measures the volume injected in a time $t$, and it is equivalent to the definition used by Gharib et al. ${ }^{1}$

A continuous $5 \mathrm{~W}$ argon-ion laser was used to illuminate the flow. The laser beam was conducted through a series of lenses and mirrors to obtain a thin laser sheet accurately
TABLE I. Summary of the experimental parameters. Here e/w (\%) is mass fraction of the ethanol/water solution.

\begin{tabular}{lccccccc}
\hline \hline Session & $\begin{array}{c}D \\
(\mathrm{~cm})\end{array}$ & $\begin{array}{c}U_{j} \\
(\mathrm{~m} / \mathrm{s})\end{array}$ & $\begin{array}{r}\mathrm{e} / \mathrm{w} \\
(\%)\end{array}$ & $\begin{array}{c}\rho_{j} \\
\left(\mathrm{~kg} / \mathrm{m}^{3}\right)\end{array}$ & $\begin{array}{c}\nu_{j} \\
\left(10^{-6} \mathrm{~m}^{2} / \mathrm{s}\right)\end{array}$ & $\mathrm{Re}$ & $\mathrm{Fr}$ \\
\hline $\mathrm{A}$ & 1.5 & 0.0732 & 0 & 999 & 1.00 & 1098 & $\infty$ \\
$\mathrm{B}$ & 1.5 & 0.1397 & 0 & 999 & 1.00 & 2095 & $\infty$ \\
$\mathrm{C}$ & 2.0 & 0.1289 & 0 & 999 & 1.00 & 2578 & $\infty$ \\
$\mathrm{D}$ & 1.5 & 0.1114 & 10 & 982 & 1.53 & 1093 & 5.0 \\
$\mathrm{E}$ & 2.0 & 0.0815 & 10 & 982 & 1.53 & 1066 & 2.0 \\
$\mathrm{~F}$ & 2.0 & 0.1296 & 75 & 853 & 2.36 & 1098 & 0.5 \\
$\mathrm{G}$ & 1.5 & 0.2028 & 30 & 954 & 2.79 & 1088 & 6.0 \\
$\mathrm{H}$ & 1.5 & 0.0865 & 10 & 982 & 1.53 & 848 & 3.0 \\
$\mathrm{I}$ & 2.0 & 0.0761 & 10 & 982 & 1.53 & 995 & 1.8 \\
$\mathrm{~J}$ & 1.0 & 0.1646 & 100 & 789 & 1.52 & 1079 & 1.0 \\
$\mathrm{~K}$ & 2.0 & 0.1463 & 65 & 877 & 2.67 & 1093 & 0.8 \\
$\mathrm{~L}$ & 1.5 & 0.1097 & 100 & 789 & 1.52 & 1079 & 0.3 \\
$\mathrm{M}$ & 2.0 & 0.0944 & 90 & 818 & 1.88 & 1002 & 0.2 \\
\hline \hline
\end{tabular}

aligned with the jet axis and parallel to two of the tank sidewalls. A high-speed camera, RedLake system X3+, was employed to record the development and evolution of the jet. Besides being used for visualization purposes, consecutive image frames were also analyzed, using high-speed particle image velocimetry (PIV) to obtain the velocity fields in a symmetry plane of the jet. The combination of the continuous laser and the high-speed camera allowed us to obtain a high temporal resolution. Images were recorded at 250 frames/s with a resolution of $1024 \times 1280$ pixels, which allowed the visualization of the jet up to ten orifice diameters downstream from the origin. However, in some experiments, where larger magnifications were required, only an area of a few diameters was imaged.

Interrogation windows of $32 \times 32$ pixels with $50 \%$ overlap were typically used in the PIV analyses performed to compute the velocity fields from the high-speed movies. This enabled measurements of velocity fields with spatial resolutions always better than $2.5 \mathrm{~mm}$, this value corresponding to the observation of ten diameters using the widest orifice. Since the PIV technique requires the presence of visible tracer particles in the flow, the water filling the tank, as well as the ethanol/water solution were seeded with neutrally buoyant, hollow glass spheres (Potters Particles Inc. of mean density of $1.1 \mathrm{~g} / \mathrm{cm}^{3}$ ).

\section{FLOW VISUALIZATIONS}

Flow visualizations of four jets with Reynolds number $\mathrm{Re}=1000$ at different Froude numbers are displayed in Fig. 2 for the same dimensionless times, namely, $t^{*}=t U_{j} / D=1,2$, 4, and 6 respectively, which expressed in terms of Eq. (4), with $t_{1} U_{j} / D=2$, correspond to $\hat{t}=0.25,1,3$, and 5 . The visualizations were obtained using a laser sheet as light source and recording the light scattered by the seeding particles introduced in the jet. A high contrast was obtained by increasing the concentration of particles in the jet with respect to 

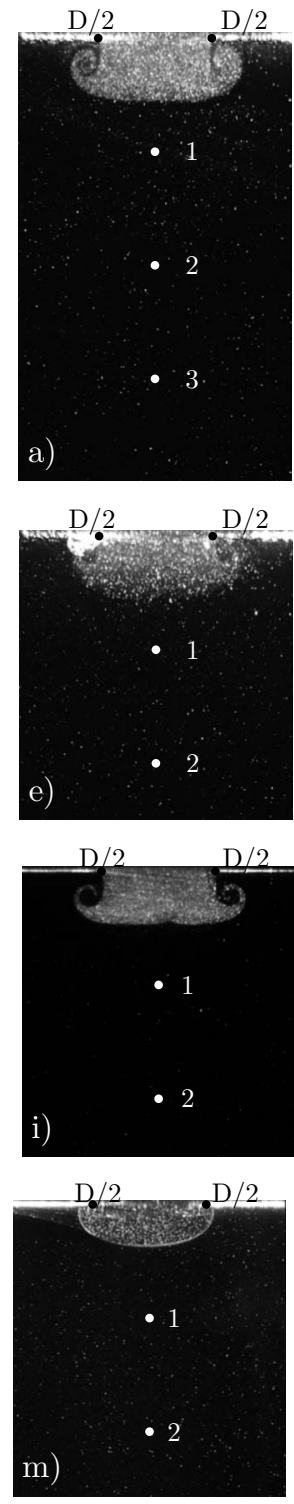
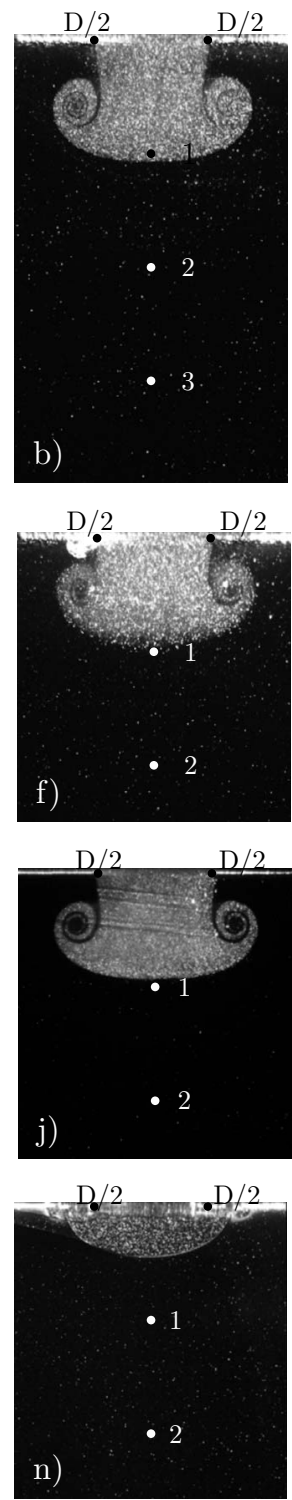
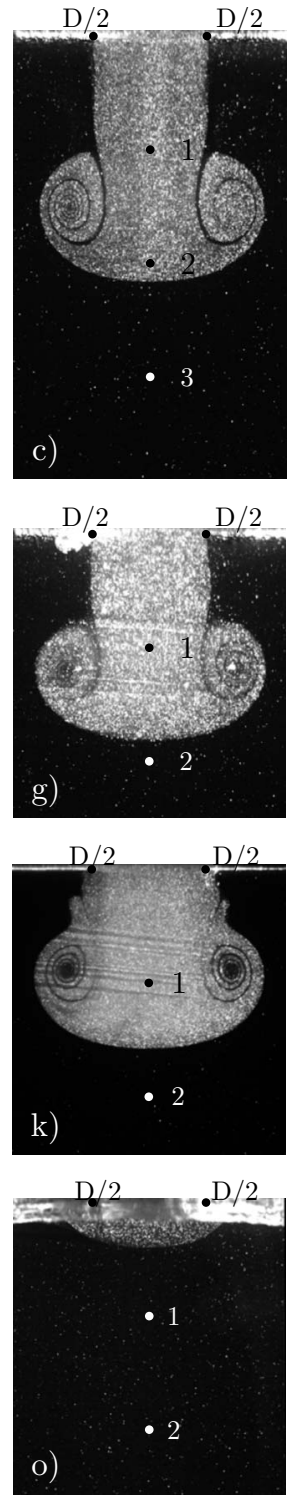
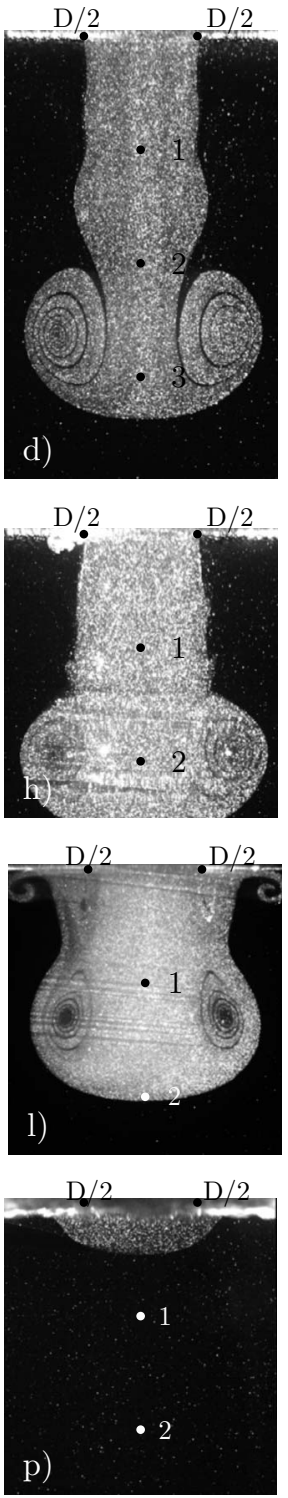

FIG. 2. Flow visualizations at $\hat{t}=0.25,1,3$, and 5 , respectively. First row [(a)-(d)] corresponds to $F r=\infty$, second [(e)-(h)], third [(i)-(l)], and fourth [(m)-(p)] to $\mathrm{Fr}=5, \mathrm{Fr}=2$, and $\mathrm{Fr}=0.5$, respectively (experiments $\mathrm{A}, \mathrm{D}, \mathrm{E}$, and $\mathrm{F}$ in Table I). The diameter of the orifice is marked, as well as the axial distance measured in diameters. To visualize the jet a higher particle seeding concentration was introduced into the piston. The jet Reynolds number is approximately 1000 in the four cases.

that of the ambient fluid. In addition, the slightly different refraction indices of the ethanol/water mixtures helped to delimit the jet's boundary in the images.

A visualization of a jet of density equal to that of the ambient fluid, $\mathrm{Fr}=\infty$, can be seen in the first row, Figs. 2(a)-2(d). The formation of the leading vortex and of secondary instabilities in the trailing jet can be observed as the vortex head moves downward. In this case, all the snapshots show the heading vortex still connected to the jet, although the starting point of the pinch-off process can be inferred in Fig. 2(d). The following rows of Fig. 2 correspond to visualizations of jets of fluid lighter than the ambient injected downward (negatively buoyant jets). Figures 2(e)-2(1) correspond to $\mathrm{Fr}=5$ and $\mathrm{Fr}=2$, respectively, whereas Figs. 2(m) $-2(p)$ correspond to a jet with a smaller Froude number, $\mathrm{Fr}=0.5$. Two different phenomena may occur depending on the Froude number: For large values of Fr the jet is formed with a vortex ring at its front, whereas for small values of $\mathrm{Fr}$, no jet is formed at all. Note that at Froude numbers $\mathrm{Fr}=5$ [Figs. 2(e) $-2(\mathrm{~h})$ ] and $\mathrm{Fr}=2$ [Figs. 2(i) $-2(\mathrm{l})$ ], the formation of the leading vortex ring, similar to the case of the homogeneous jet, can be easily identified. However, comparing to the case of $\mathrm{Fr}=\infty$, in these cases the jet moves slower, and it may even stop before the axial symmetry is lost. Nevertheless the existence of a vortex ring at the front of the jet is clear. Another interesting aspect is the production of small vortices of lighter fluid that are convected toward the top of the tank. Observe one of these vortices, clearly identified on the top wall in Fig. 2(1), moving radially outward. Again the appearance of secondary trailing vortices in the jet is evident. Furthermore, it has been noticed in some cases an oscillatory motion around the maximal penetration depth, as described by Turner, ${ }^{8}$ while in other cases the axial symmetry was lost before it happened. 
TABLE II. Summary of the combination of Reynolds and Froude numbers of the numerical simulations performed. Here e/w (\%) is mass fraction of the ethanol/water solution of the jet.

\begin{tabular}{lrcl}
\hline \hline Session & $\begin{array}{r}\text { e/w } \\
(\%)\end{array}$ & Re & Fr \\
\hline i & 0 & 2000 & $\infty$ \\
ii & 0 & 1000 & $\infty$ \\
iii & 10 & 2034 & 7 \\
iv & 3 & 1007 & 7 \\
v & 10 & 1308 & 3 \\
vi & 4 & 990 & 2.4 \\
vii & 10 & 1007 & 1.8 \\
viii & 86 & 2007 & 1 \\
ix & 91 & 2021 & 0.8 \\
x & 100 & 1908 & 0.4 \\
xi & 100 & 1230 & 0.4 \\
\hline \hline
\end{tabular}

If the Froude number is small enough [see Figs. $2(m)-2(p)]$, the vortex ring produced at the beginning of the piston motion is not able to travel downward and remains close to the wall, forming a caplike type of flow. The formation of this vortex ring is clear in the visualizations and it can be detected with PIV analysis as well as in the numerical simulations, as it will be shown in Sec. V. The vortex ring is not able to penetrate into the ambient due to the buoyancy forces, and it is pushed toward the top wall, where it begins to move radially as a second vortex forms at the front position. So as in the cases described above, there is also vortex shedding from the vortex head. As the injection of fluid from the cylinder continues, the flow spreads radially and the vortices travel near the top wall.

As a summary, there seems to exist a critical Froude number, $\mathrm{Fr}_{c}$, that distinguishes two different flow regimes: a regime at $\mathrm{Fr}>\mathrm{Fr}_{c}$ where it can be observed the formation of a vortex ring followed by a trailing jet and a regime at $\mathrm{Fr}<\mathrm{Fr}_{c}$ where no jet is formed at all. This critical Froude number has a value close to unity, as described later on in Sec. V.

\section{NUMERICAL SIMULATIONS}

To perform the numerical simulations the unsteady, laminar Navier-Stokes equations in cylindrical coordinates were integrated using a finite volume technique. The energy equation was not used since the temperature was not included in our study and hence, only the continuity, Eq. (5), momentum, Eqs. (6) and (7), and species conservation, Eq. (8), equations were solved,

$$
\frac{\partial \rho}{\partial t}+\frac{\partial}{\partial x}\left(\rho u_{x}\right)+\frac{1}{r} \frac{\partial}{\partial r}\left(\rho u_{r} r\right)=0,
$$

$$
\begin{aligned}
& \frac{\partial}{\partial t}\left(\rho u_{x}\right)+\frac{\partial}{\partial x}\left(\rho u_{x} u_{x}\right)+\frac{1}{r} \frac{\partial}{\partial r}\left(\rho r u_{r} u_{x}\right) \\
& =-\frac{\partial p}{\partial x}+\frac{\partial}{\partial x}\left[\mu\left\{2 \frac{\partial u_{x}}{\partial x}-\frac{2}{3}\left[\frac{\partial u_{x}}{\partial x}+\frac{1}{r} \frac{\partial\left(r u_{r}\right)}{\partial r}\right]\right\}\right] \\
& +\frac{1}{r} \frac{\partial}{\partial r}\left[r \mu\left(\frac{\partial u_{r}}{\partial x}+\frac{\partial u_{x}}{\partial r}\right)\right]-\left(\rho_{0}-\rho\right) g \text {, } \\
& \frac{\partial}{\partial t}\left(\rho u_{r}\right)+\frac{\partial}{\partial x}\left(\rho u_{r} u_{x}\right)+\frac{1}{r} \frac{\partial}{\partial r}\left(\rho r u_{r} u_{r}\right) \\
& =-\frac{\partial p}{\partial r}+\frac{\partial}{\partial x}\left[\mu\left(\frac{\partial u_{r}}{\partial x}+\frac{\partial u_{x}}{\partial r}\right)\right]+\frac{1}{r} \frac{\partial}{\partial r} \\
& \times\left[r \mu\left\{2 \frac{\partial u_{r}}{\partial x}-\frac{2}{3}\left[\frac{\partial u_{x}}{\partial x}+\frac{1}{r} \frac{\partial\left(r u_{r}\right)}{\partial r}\right]\right\}\right]-2 \mu \frac{u_{r}}{r^{2}} \\
& +\frac{2}{3} \frac{\mu}{r}\left[\frac{\partial u_{x}}{\partial x}+\frac{1}{r} \frac{\partial\left(r u_{r}\right)}{\partial r}\right] \text {, } \\
& \frac{\partial}{\partial t}(\rho X)+\frac{\partial}{\partial x}\left(\rho u_{x} X\right)+\frac{\partial}{\partial r}\left(\rho u_{r} X\right) \\
& =\frac{\partial}{\partial x}\left(\rho \Phi \frac{\partial X}{\partial x}\right)+\frac{1}{r} \frac{\partial}{\partial r}\left(\rho \Phi r \frac{\partial X}{\partial r}\right) .
\end{aligned}
$$

In Eqs. (5)-(8) $u_{x}$ and $u_{r}$ are the axial and radial components of the velocity, $\rho(t, x, r)$ and $\mu(t, x, r)$, which depend on time, $t$, as well as on the axial, $x$, and radial position, $r$, are the density and viscosity of the ethanol/water solution, $\Phi$ is the diffusion coefficient, and $X$ is the mass fraction of one of the fluids.

A first order scheme was used to model the temporal terms, and the time step was dynamically changed based on the truncation error associated with the temporal integration scheme. The convective derivatives were modeled with a segregated method and a second-order implicit scheme whereas for the diffusion terms a central-differenced secondorder accurate scheme was used. The gravitational term was not negligible and, consequently, had to be also implemented. A scheme from the family of the SIMPLE algorithms was used for the coupling of pressure and velocity.

The numerical domain consisted of a rectangular grid of size $10 D$ in the radial direction and $20 D$ in the axial direction: The simulations were stopped long before the front of the jet reached any of the boundaries. An initial grid of more than 30000 cells, especially refined near the jet exit was dynamically adapted as the jet developed using a pressure gradient criterion. Tests were performed to check that the grid was suitable for this problem by mesh refinement (the same method was also used to test the suitability of the time step). Table II summarizes the characteristic dimensionless parameters of the numerical simulations reported in this work.

At time $t=0$ the fluid was considered to be at rest with density, $\rho_{0}$, and viscosity, $\mu_{0}$, specified for the ambient fluid. Previous studies have focused on the effects of the velocity profile and the time evolution of the piston velocity at the jet exit on the vorticity and on the total circulation of the 


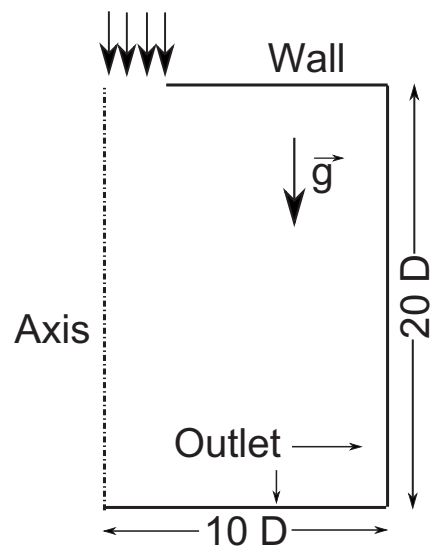

FIG. 3. Sketch of the numerical domain used in the simulations.

jet. ${ }^{5,6,14}$ In our simulations, to clearly identify the effects of negative buoyancy from any other parameters, we imposed a uniform and time-independent velocity profile at the injection orifice, $U_{j}$. The density, $\rho_{j}$, and the viscosity, $\mu_{j}$, were determined using a polynomial fit to the experimental data collected by Weast ${ }^{15}$ to take into account their nonlinear behavior of the fluid properties when ethanol and water are mixed. The diffusion coefficient of ethanol in water obtained from Perry and Green ${ }^{16}$ was also used in the calculations, $\Phi=1.28 \times 10^{-9} \mathrm{~m}^{2} / \mathrm{s}$. To check the relevance of having a nonlinear dependence of the mixture properties with the ethanol concentration, we compared the results obtained from numerical simulations performed using the real functions of density and viscosity with those given using linearly varying properties. However, the results obtained in both cases were nearly identical on the time scales of interest.

A sketch of the numerical domain with the boundary conditions imposed can be observed in Fig. 3. Nonslip velocity was fixed at the upper boundary (wall) and static pressure was imposed at the boundary opposed to the jet exit and at the lateral boundary (outlet). Finally symmetry conditions were imposed on the axis (symmetry).

\section{EXPERIMENTAL AND NUMERICAL RESULTS}

The most important parameters of negatively buoyant jets have been obtained experimentally through the analysis of the acquired images and compared to the simulations performed with the numerical method described above.

\section{A. Front position and penetration depth}

The jet front position, defined as the position of the head of the vortex, was automatically obtained from the experimental images, using a gray threshold. All the experiments displayed in Fig. 4 were carried out for different Froude numbers keeping the Reynolds number close to 1000 . Note that a steady state is reached for experiments with small Froude number, $\mathrm{Fr}<1$, for which the final penetration depth remains constant. Generally, this happens after the transient regime, where the maximum penetration depth is achieved before the jet drops to the constant depth. For intermediate Froude numbers, $1<\mathrm{Fr}<3$, no stationary penetration dis-

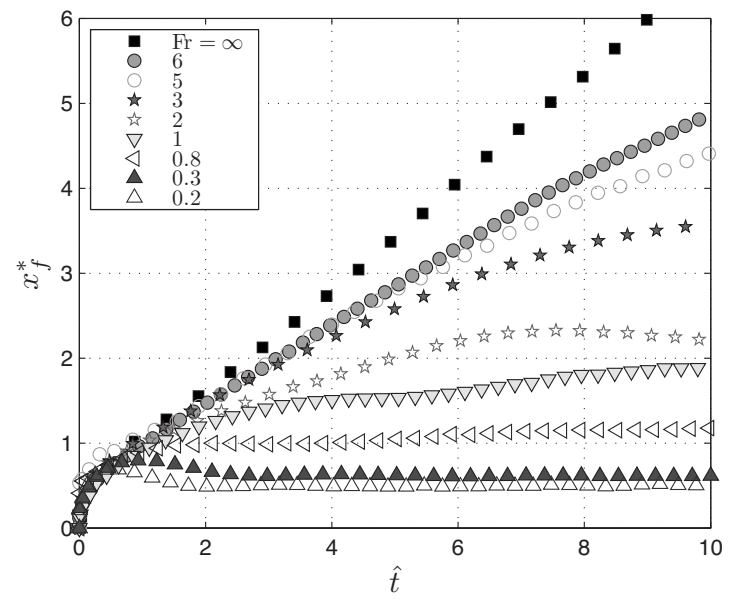

FIG. 4. Experimental measurements of the time evolution of the front position for different Froude numbers, at $\mathrm{Re} \approx 1000(\mathrm{Fr}=\infty$ corresponds to experiment $\mathrm{A}$ in Table $\mathrm{I}, \mathrm{Fr}=6$ to $\mathrm{G}, \mathrm{Fr}=5$ to $\mathrm{D}, \mathrm{Fr}=3$ to $\mathrm{H}, \mathrm{Fr}=2$ to $\mathrm{E}, \mathrm{Fr}=1$ to $\mathrm{J}, \mathrm{Fr}=0.8$ to $\mathrm{K}, \mathrm{Fr}=0.3$ to $\mathrm{L}$, and $\mathrm{Fr}=0.2$ to $\mathrm{M}$ ).

tance is reached: For times longer than those represented in Fig. 4 the front oscillated around a fixed position before loosing its symmetry. Finally, for larger Froude numbers the imaged area was too small to observe this behavior.

Figure 5 summarizes the final penetration depth (steady or oscillatory) obtained by different authors ${ }^{10,17-21}$ in a wide range of Froude numbers. This plot is based on a similar figure published by Kaye and $\mathrm{Hunt}^{17}$ compiling results available in literature, where we have included our measurements for comparison. The different definitions of the Froude number used by other authors have been adequately expressed in terms of the Froude number used in this work. Three different regimes can be clearly identified: For very low Froude numbers the steady penetration depth scales as $x_{\mathrm{st}}^{*} \sim \mathrm{Fr}^{1 / 3}$. However, in an intermediate regime, where our subcritical experiments lie, the dependence changes to a $x_{\mathrm{st}}^{*} \sim$ Fr law.

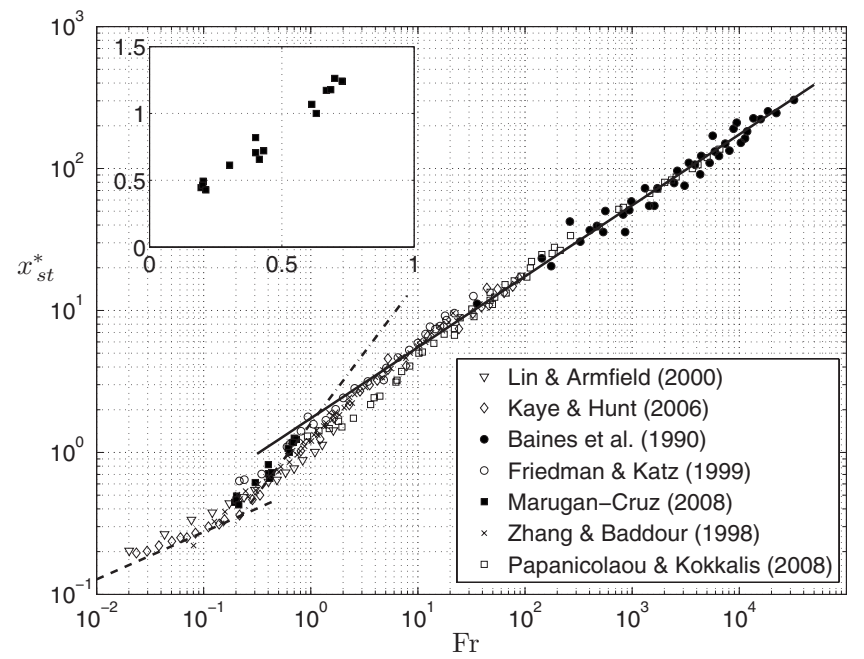

FIG. 5. Dependence of the penetration depth with the Froude number reported by different authors (Refs. 10 and 17-21) along with our measurements obtained at $\operatorname{Fr}<1$. The inset shows a detail of our measurements to indicate the linear dependence of $x_{\mathrm{st}}^{*}$ with Fr. Here dashed line corresponds to a power law $x_{\mathrm{st}}^{*} \sim \mathrm{Fr}^{1 / 3}$, dashed-dotted to $x_{\mathrm{st}}^{*} \sim \mathrm{Fr}$, and solid line to $x_{\mathrm{st}}^{*} \sim \mathrm{Fr}^{1 / 2}$. 

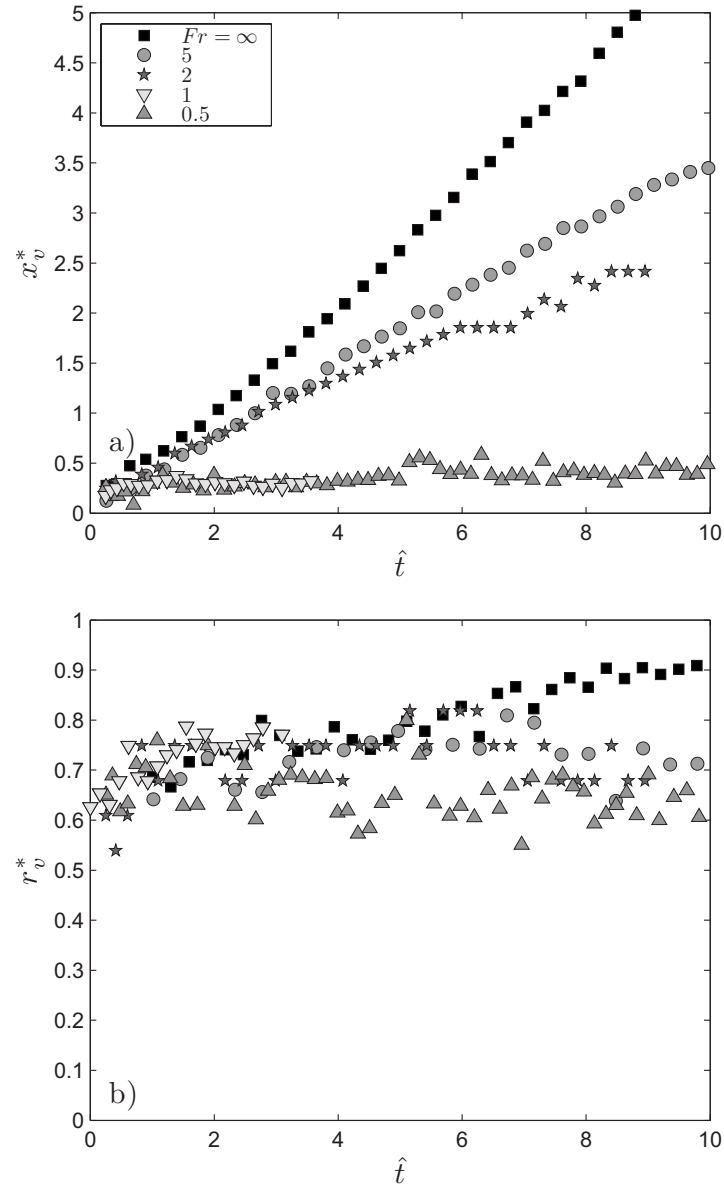

FIG. 6. Experimental measurements of the leading vortex position for different Froude numbers: (a) axial position and (b) radial position $(\mathrm{Fr}=\infty$ corresponds to experiment A in Table $\mathrm{I}, \mathrm{Fr}=5$ to $\mathrm{D}, \mathrm{Fr}=2$ to $\mathrm{E}, \mathrm{Fr}=1$ to $\mathrm{J}$, and $\mathrm{Fr}=0.5$ to $\mathrm{F}$ ).

Finally, for Fr high enough the final penetration depth scales as $x_{\mathrm{st}}^{*} \sim \mathrm{Fr}^{1 / 2}$, although it should be pointed out that in this latter regime no steady position is reached, and the results correspond to the mean value around which the jet front oscillates. We have also represented as an inset in this figure the stationary penetration depth versus the Froude number obtained from our measurements at $\mathrm{Fr}<1$, to show its linear dependence, $x_{\mathrm{st}}^{*} \sim$ Fr. Notice that our results agree with those reported by Kaye and Hunt, ${ }^{17}$ Friedman and Katz, ${ }^{18}$ and Zhang and Baddour ${ }^{19}$ in the same range of Froude numbers. However, no evidence has been found of any relationship between the penetration depth and the Reynolds number for the cases studied here.

\section{B. Vortex head position}

The leading vortex location was calculated as the point of maximum vorticity (the details of the vorticity calculation are given in Sec. V C). To locate the vorticity peak, an estimation of fractional displacement method, which consists of a parabolic-fit estimator, ${ }^{22}$ was used.

Like the penetration depth, the axial position of the leading vortex, $x_{v}^{*}$, is strongly influenced by the Froude number [see Fig. 6(a)], not only because the maximum axial position corresponds to the largest Froude number but also because the velocity of the vortex ring decreases with time faster when the Froude number decreases. On the contrary, the final radial position, $r_{v}^{*}$, is soon reached in all cases, meaning that the vortex is not growing radially, as shown in Fig. 6(b). Thus, the dependence of the radial position of the leading vortex with the Froude number is relatively small compared to that of the axial position. It is important to notice that the axial and radial locations of the vortex ring in the case of the smallest Froude number shown in this figure, $\mathrm{Fr}=0.5$, does not refer to the initial vortex formed when the piston begins to move, but to the vortex that, at each instant of time, is in front of the jet.

\section{Vorticity fields}

A five point least-square method, proposed by Raffel et al. $^{23}$ and Acosta-Iborra, ${ }^{24}$ was used to calculate the out-ofplane dimensionless vorticity from the velocity measurements for the cases where the characteristic size of the vortex was sufficiently large,

$$
\omega^{*}=(\nabla \wedge \vec{u})_{\theta} D / U_{j}=\left(\frac{\partial u_{r}}{\partial x}-\frac{\partial u_{x}}{\partial r}\right) \frac{D}{U_{j}} .
$$

Figure 7 shows the experimental dimensionless vorticity contours of the jets displayed in Fig. 2. Figures 7(a)-7(d) show the evolution of the out-of-plane vorticity for the homogeneous jet. The formation of the vortex head can be observed during the initial instants followed by the generation of secondary vortices as time evolves. During the time that the vortex head has been tracked, the vortex ring remains attached to the trailing jet and, consequently, there is a continuous injection of vorticity from the jet into the leading vortex. As described by Carter et al., ${ }^{25}$ the vortex ring has an initial round shape but as it engulfs more vorticity its shape changes toward a more elliptic one.

The vorticity contours of the jet at $\mathrm{Fr}=5$ are displayed in Figs. $7(\mathrm{e})-7(\mathrm{~h})$. Notice that initially the vorticity distribution is similar to that of the homogeneous jet, however, at longer times, it is observed a faster formation of the secondary instabilities and a more concentrated distribution of vorticity in the trailing jet. Another phenomenon that takes place as time evolves is the generation of a vorticity layer of negative sign surrounding the vortex ring. Since this layer contains fluid of density lower than that of the ambient fluid, it is pushed upward by the buoyancy forces until it eventually reaches the top wall. It will be shown later on in Sec. VI that this negative vorticity has a baroclinic origin, and therefore we will refer to it, from now on, as baroclinic vorticity.

The evolution of vorticity for a jet at $\mathrm{Fr}=2$ has been represented in Figs. 7(i)-7(1). As in the case of Fr=5, during the initial times while the vortex ring is being formed, the vorticity contours are similar to those for $\mathrm{Fr}=\infty$. However, at larger times several differences can be observed: (1) as was already described above, the vortex head moves slower than in the homogeneous case and, more importantly, (2) the baroclinic vorticity layer, of opposite sign to that of the vortex head, clearly develops at the outside of the jet. This layer is generated at the front of the leading vortex and travels 

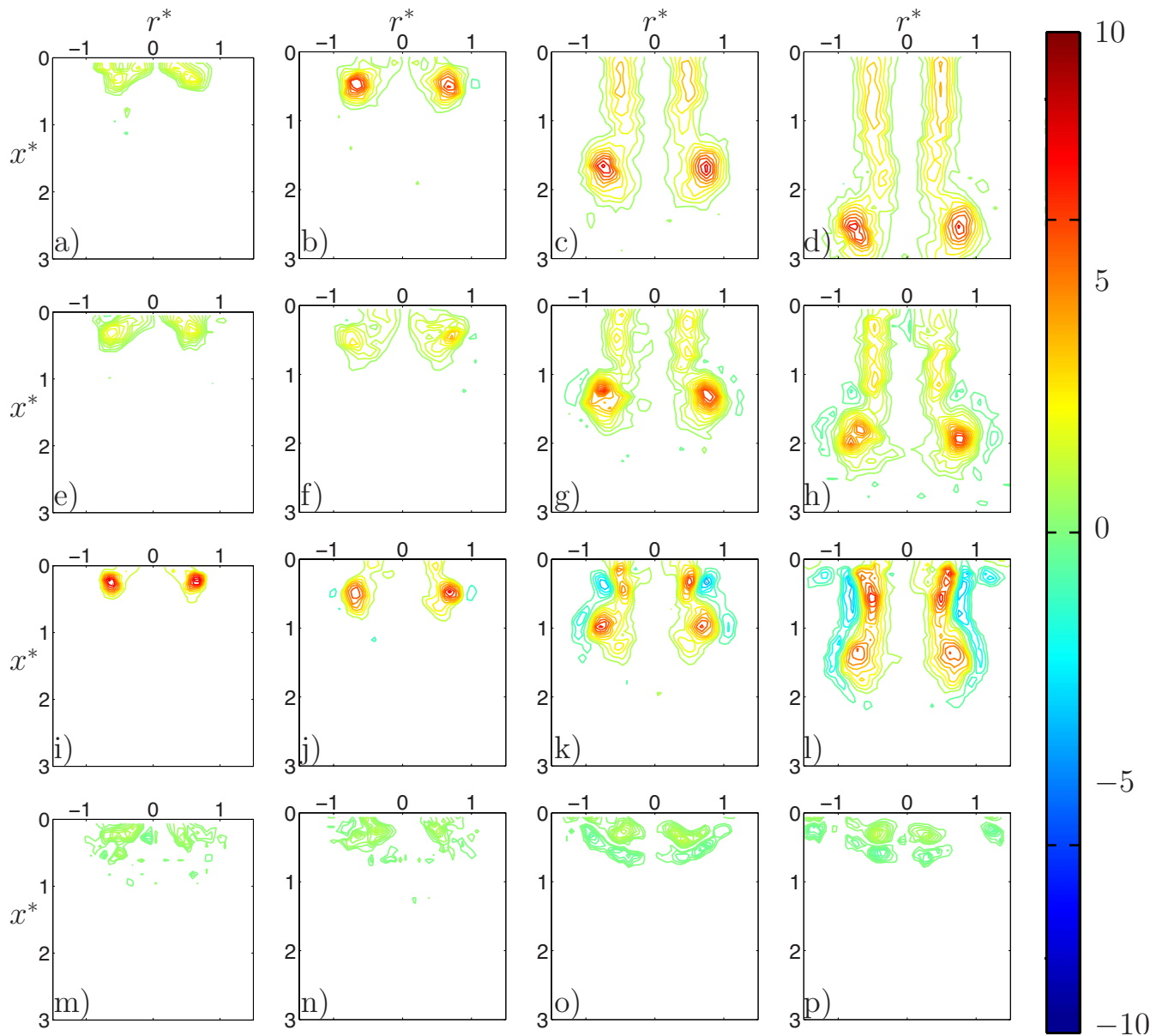

FIG. 7. (Color online) Vorticity contours for $\hat{t}=0.25,1,3$, and 5. First row [(a)-(d)] corresponds to $\mathrm{Fr}=\infty$, second [(e)-(h)], third [(i)-(1)], and last row $[(\mathrm{m})-(\mathrm{p})]$ to $\mathrm{Fr}=5, \mathrm{Fr}=2$, and $\mathrm{Fr}=0.5$, respectively (experiments A, D, E, and F in Table I).

upstream faster than the vortex head itself, reaching the top of the tank and expanding radially along the upper wall.

Finally, the extreme case is shown in Figs. 7(m)-7(p) for $\mathrm{Fr}=0.5$. In this case, the vortex head has no time to fully develop before it is pushed away in the radial direction. Meanwhile another vortex ring is formed which will not be able to fully develop either and the same process is repeated over and over.

In addition to the experimental measurements of the vorticity fields described above, the vorticity was also calculated from the velocity fields obtained in the numerical simulations, as $\omega_{\theta}=\left(\partial u_{r} / \partial x-\partial u_{x} / \partial r\right)$. The vorticity contours of four jets with $\mathrm{Re} \simeq 1000$ and different Froude numbers are shown in Fig. 8 at the same nondimensional times as in Fig. 7.

The evolution of the vorticity of a homogeneous jet, $\mathrm{Fr}=\infty$, has been represented in Figs. 8(a)-8(d). The formation of the vortex head during the initial instants, followed by the jet, can be seen as time evolves. The vortex ring remains attached to the trailing jet and, consequently, there is a continuous injection of circulation from the jet into the leading vortex. Since the flow was not artificially perturbed there are no secondary instabilities in any of the cases.

The appearance of the baroclinic vorticity is clear in the vorticity fields of the lighter jets. In Figs. 8(e)-8(h) the vorticity fields of a jet with $\mathrm{Fr}=7$ have been plotted. The baroclinic vorticity layer surrounding the leading vortex ring is clear at the largest time shown, $\hat{t}=5$. In Figs. 8(i)-8(1) this vorticity layer of opposite sign is identified at shorter times, and even at an earlier stage it can be visualized in the case of the subcritical jet [Figs. 8(m)-8(p)].

\section{Total circulation, vortex circulation, and formation number}

The total circulation was calculated as the integral of the axial velocity along the integration domain as

$$
\Gamma^{*}=\frac{\Gamma}{U_{j} D}=\frac{1}{U_{j} D} \oint_{C} \vec{u} \cdot \mathrm{d} \vec{l}=\frac{1}{U_{j} D} \int_{0}^{\infty} u_{x}(r=0, x) \mathrm{d} x,
$$

where $C$ is the curve enclosing surface $S_{0}$ (see Fig. 15).

Several experiments were carried out in order to determine the influence of the Froude number on the total circulation. Figure 9 represents the time evolution of the dimensionless total circulation of the jet for different values of the Froude number obtained experimentally as well as from the numerical simulations. The differences between the numerical results and the experiments are mainly caused by the different velocity profiles at the exit of the orifice, as well as by the different time evolutions of the piston velocity in our experiments. The important aspect shown in Fig. 9 is the different evolution of $\Gamma^{*}$ as the Froude number decreases. In 


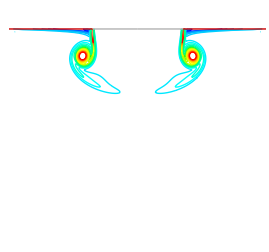

a)

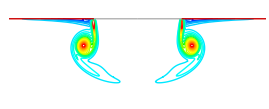

e)

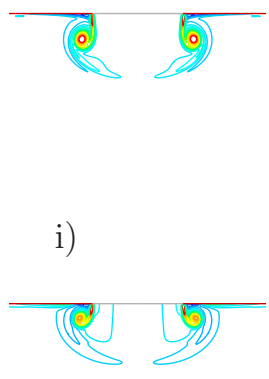

m)

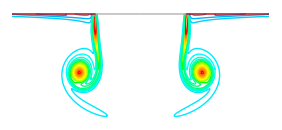

b)

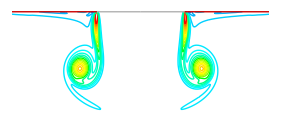

f)

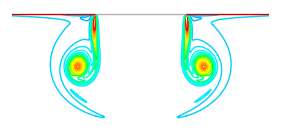

j)

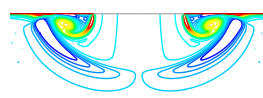

n)

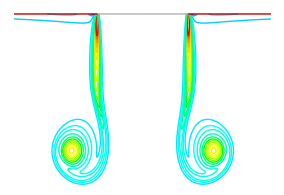

c)

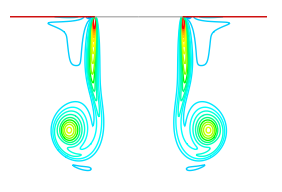

g)

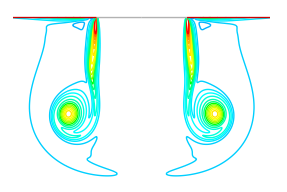

k)

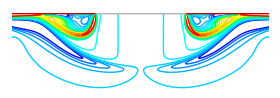

o)

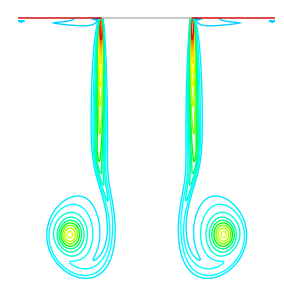

d)

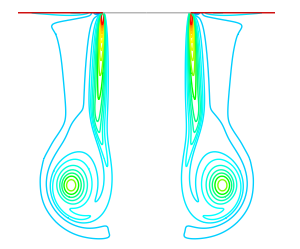

h)

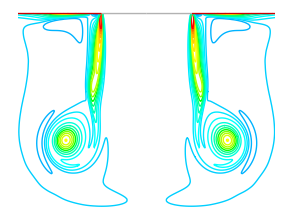

l)

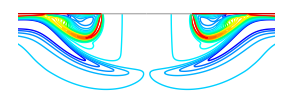

p)
20

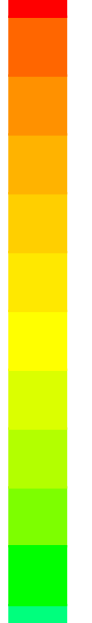

10

0

$-5$

FIG. 8. (Color online) Numerical vorticity contours for $\hat{t}=0.25,1,3$, and 5. First row [(a)-(d)] corresponds to $\operatorname{Fr}=\infty$, second [(e)-(h)], third [(i)-(1)], and last row $[(\mathrm{m})-(\mathrm{p})]$ to $\mathrm{Fr}=7, \mathrm{Fr}=3$, and $\mathrm{Fr}=0.4$, respectively (numerical simulations ii, iv, $\mathrm{v}$, and xi of Table II). Note that here $\hat{t}=t^{*}$ since our numerical simulations were started impulsively.

fact, it can be observed that at $\mathrm{Fr}<1$ the total circulation reaches a maximum at $\hat{t}=1$ and later on decreases toward a constant value of approximately $\Gamma^{*}=0.5$.

Another interesting characteristic of starting jets is the formation number, $F$, defined as the dimensionless time,

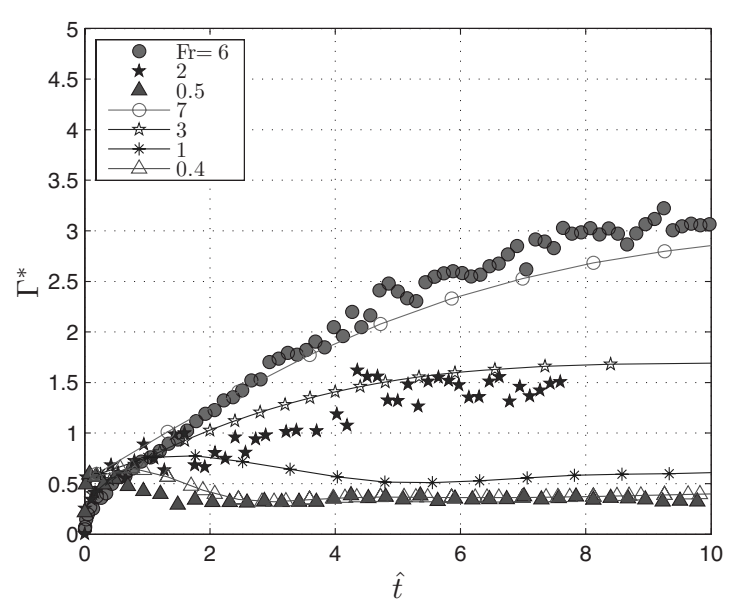

FIG. 9. Time evolution of the total circulation of the jet for different Froude numbers. The hollow symbols correspond to the numerical simulations ( $\mathrm{Fr}=7$ corresponds to numerical simulation iii, $\mathrm{Fr}=3$ to $\mathrm{v}, \mathrm{Fr}=1$ to viii, and 0.4 to $\mathrm{x}$ in Table II); the solid symbols represent the experimental results [experiments $\mathrm{G}(\mathrm{Fr}=6), \mathrm{E}(\mathrm{Fr}=2)$, and $\mathrm{F}(\mathrm{Fr}=0.5)$ in Table I].
$F=t_{f} U_{j} / D$, at which the circulation injected by the discharging jet is equal to the circulation that the vortex head will have once it eventually detaches. ${ }^{1}$ As already mentioned, to calculate the circulation of the vortex ring, it must be disconnected from the trailing jet. Consequently, we considered the pinch-off time to be the instant at which the vorticity contour line $\omega^{*}=1$ enclosed the vortex core, and estimated the vortex circulation as the circulation inside that contour line. Previous studies have shown that for neutrally buoyant jets, the formation number, $F$, can vary depending on the velocity profile and the time evolution of the piston velocity. ${ }^{5,6,14}$ For our experimental setup the formation number for $\mathrm{Fr}=\infty$ varied between 2.5 and 3.5 as can be deduced from Fig. 10. This figure also shows that the evolution of the total circulation of the jet does not depend on the Reynolds number, at least, in the range of Reynolds numbers studied in this work.

In the case of light jets, $\operatorname{Fr}<\infty$, one would expect the leading vortex to have a higher circulation since it disconnects from the trailing jet later than in the case of homogeneous jet; however, we have observed a different behavior. Figure 11 compares both the total and the vortex circulation of two jets with identical Reynolds number, $\mathrm{Re}=2000$, and different Froude numbers, i.e., $\mathrm{Fr}=\infty$ and Fr=7. The dasheddotted lines indicate the graphical procedure used to determine the formation time. Note that the vortex circulation, 


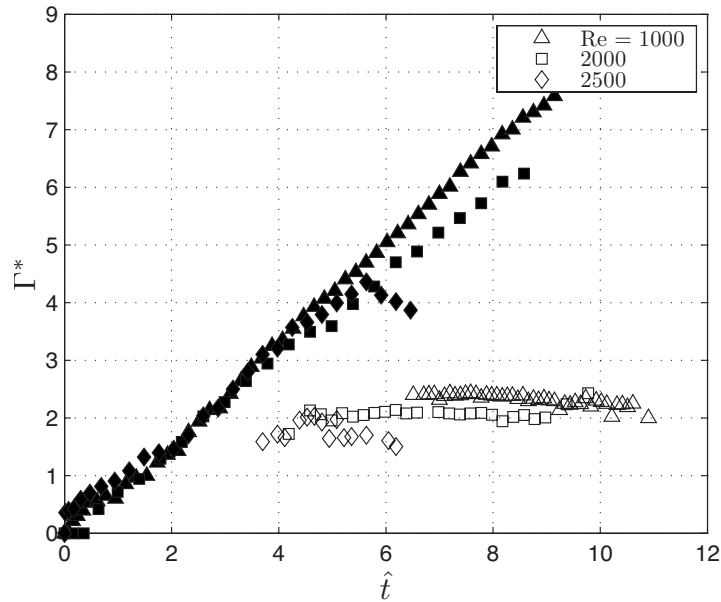

FIG. 10. Total and vortex circulation for three jets of $\mathrm{Fr}=\infty$ and different Reynolds numbers (experiments A, B, and C in Table I). The solid symbols represent the total circulation and the hollow symbols the vortex circulation.

and consequently the formation number, is substantially smaller in the negatively buoyant jet $[F(\mathrm{Fr}=\infty)=3$ versus $F(\operatorname{Fr}=7)=1]$.

For jets of Froude number smaller than a critical value close to unity, $\mathrm{Fr}<\mathrm{Fr}_{c} \sim 1$, the formation number has no meaning since there is no trailing jet and the vortex head is connected to the emerging fluid because of the buoyancy forces [see Fig. 2(p)].

\section{DISCUSSION}

Observation of the visualizations and vorticity contours shown in Secs. III and V suggests the existence of two different flow regimes: (1) a first regime where the leading vortex ring does not have time to develop before it is pushed radially away from the vorticity layer and (2) a second regime where a trailing jet forms before the vortex ring reaches the maximum penetration depth. The transition between

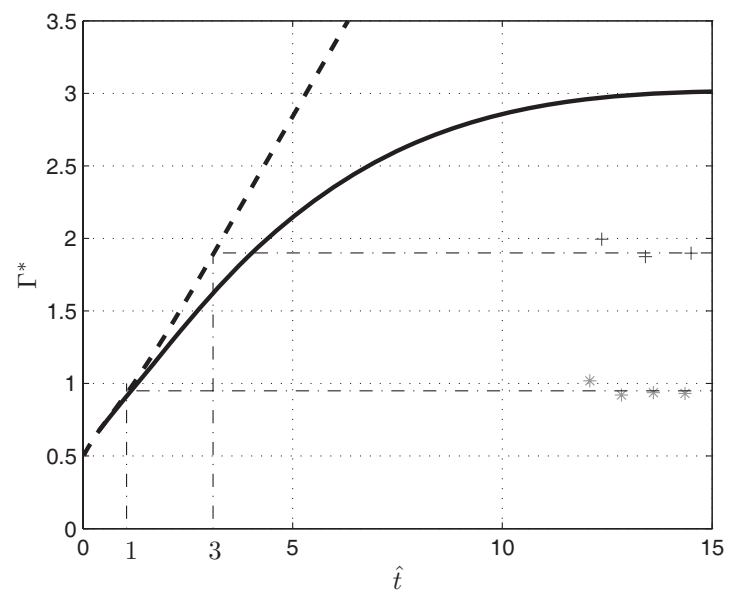

FIG. 11. Total and vortex circulation of two jets of $\mathrm{Fr}=\infty$ and $\mathrm{Fr}=7$ respectively, both with $\operatorname{Re}=2000$, obtained from the numerical simulations (numerical simulations i and iii in Table II). Dashed line corresponds to the total circulation of a homogeneous jet $\mathrm{Fr}=\infty$ and solid line to a jet of $\mathrm{Fr}=7$. Here $(+)$ represents the vortex circulation of the homogeneous leading vortex ring and $(*)$ that of the lighter one. Dashed-dotted lines illustrate the graphical procedure used to determine the formation number. these two regimes seems to take place around a critical Froude number of $\mathrm{Fr}_{c} \sim 1$. However, the mere examination of the visualizations is not sufficient to provide a quantitative characterization of what changes in the flow field when the transition occurs. In what follows, the trajectories of the vortex centers are analyzed with the aim of characterizing this transition and providing a clear definition of both subcritical and supercritical flows.

\section{A. Vortex location}

A close examination of Fig. 6 reveals that at short times, $\hat{t} \leq 1$, the axial vortex position does not depend on the Froude number, indicating that buoyancy effects are nearly negligible during the initial instants of time. Indeed, it is reasonable to assume that buoyancy effects will not take place until the acceleration due to the reduced gravity, $g^{*}=\Delta \rho g / \rho_{j}$, has time to induce a velocity comparable to the jet injection velocity, $U_{j}$. Thus, we can define a gravitational time, $t_{g}$, as

$$
t_{g}=\frac{U_{j}}{g^{*}}=\frac{\rho_{j} U_{j}}{g \Delta \rho}=\operatorname{Fr} \frac{D}{U_{j}} .
$$

In other words, the effect of the reduced gravity will become important after a dimensionless time of the order of the Froude number, $t^{*} \sim t_{g}^{*}=$ Fr. With this idea in mind, we can decompose the vortex location for any Froude number at a given time as the sum of the vortex location for a homogeneous jet of $\mathrm{Fr}=\infty, x_{v(\infty)}$, plus a term that accounts for the displacement caused by gravity, $x_{v}=x_{v(\infty)}+x_{v, g^{*}}$. Moreover, if the velocity induced by gravity at a time $t$ is of the order $g^{*} t$, the leading vortex of a light jet will deviate from the position of the leading vortex of a homogeneous jet a distance $x_{v, g^{*}} \sim-1 / 2 g^{*} t^{2}$, the minus sign indicating that gravity opposes the momentum flux in the case of light jets. Thus, the vortex location can finally be given by $x_{v}=x_{v(\infty)}-\frac{1}{2} \alpha g^{*} t^{2}$, which can be expressed in dimensionless form as

$$
\frac{x_{v(\infty)}^{*}-x_{v}^{*}}{\operatorname{Fr}}=\frac{1}{2} \alpha\left(\frac{t^{*}}{\mathrm{Fr}}\right)^{2},
$$

where $\alpha$ is a proportionality constant of order unity. To confirm this conjecture, the left hand side of Eq. (12) has been plotted versus the dimensionless time $\hat{t} / \mathrm{Fr}$ in Fig. 12. It can be shown in this figure that the numerical results collapse remarkably well onto a parabola $(\hat{t} / \mathrm{Fr})^{2}$ as the model provided in Eq. (12) predicts. Here, in the numerical simulations $\hat{t}=t^{*}$ since the flow is impulsively started, whereas the initial piston acceleration has been taken into account in the experimental measurements. Notice that although the experimental results seem to follow the same trend for $\hat{t} / \mathrm{Fr} \gtrsim 2$, some disagreement is observed at shorter times, presumably due to the effect of the velocity profile and the perturbations in the flow.

Moreover, the time evolution of the axial position of the leading vortex obtained from the numerical simulations for different Froude numbers has also been plotted in Fig. 13. It is interesting to point out that for the subcritical cases, $\mathrm{Fr}<1$, all the time evolutions of the axial vortex position, scaled with the Froude number, $x_{v}^{*} / F r$, collapse onto a single 


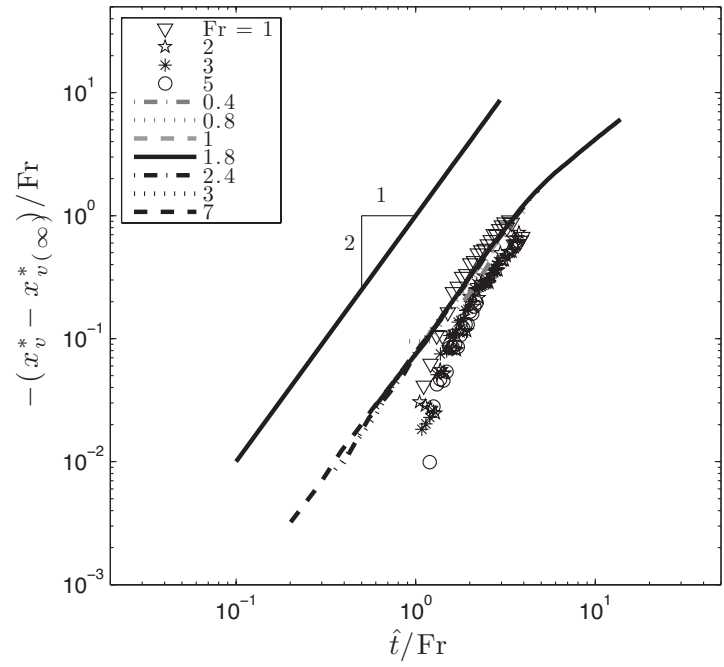

FIG. 12. Distance between the leading vortex ring of a neutrally buoyant jet and the vortex ring from nonhomogeneous starting jets scaled with the Froude number as a function of $\hat{t} / \mathrm{Fr}$. Lines correspond to numerical simulations $(\mathrm{Fr}=0.4$ corresponds to simulation $\mathrm{x}, 0.8$ to ix, 1 to viii, 1.8 to vii, 2.4 to vi, 3 to $\mathrm{v}$, and 7 to iii in Table II) and symbols to experimental data $(\mathrm{Fr}=1$ corresponds to experiment J, 2 to $\mathrm{E}, 3$ to $\mathrm{H}$, and 5 to $\mathrm{D}$ in Table I). The black solid line has a slope of 2 . Note that $\hat{t}=t^{*}$ in the numerical simulations.

curve when the time is as well rescaled with the Froude number, $\hat{t} / \mathrm{Fr}$. However, the time evolution of the vortex location depends on the Froude number in the supercritical cases, $\mathrm{Fr}>1$. Note also that the collapse observed at $\mathrm{Fr} \leq 1$ is only compatible with Eq. (12) provided that the vortex location for $\mathrm{Fr}=\infty$ is of the form $x_{v(\infty)}^{*}=V_{0} t^{*}$. Thus, in such cases Eq. (12) can be expressed as

$$
\frac{x_{v}^{*}}{\mathrm{Fr}}=V_{0} \frac{t^{*}}{\mathrm{Fr}}-\frac{1}{2} \alpha\left(\frac{t^{*}}{\mathrm{Fr}}\right)^{2}
$$

indicating that the leading vortex starts moving at a constant speed, $V_{0}$, independently of the Froude number.

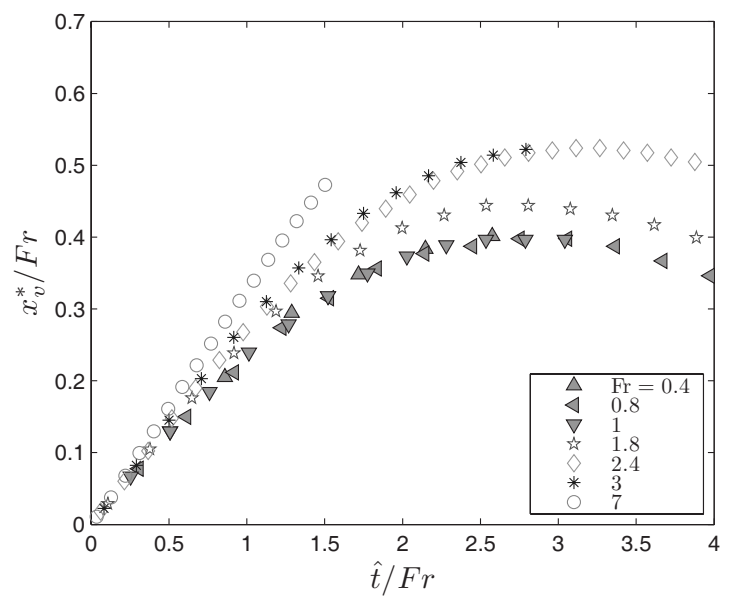

FIG. 13. Scaled axial position of the vortex head from numerical simulations. The solid symbols correspond to subcritical starting jets, $\mathrm{Fr}<1$, and the hollow symbols to supercritical, $\mathrm{Fr}>1$ (the numerical simulations are those of Fig. 12). Note that here $\hat{t}=t^{*}$ since in our numerical simulations $t_{1}=0$.

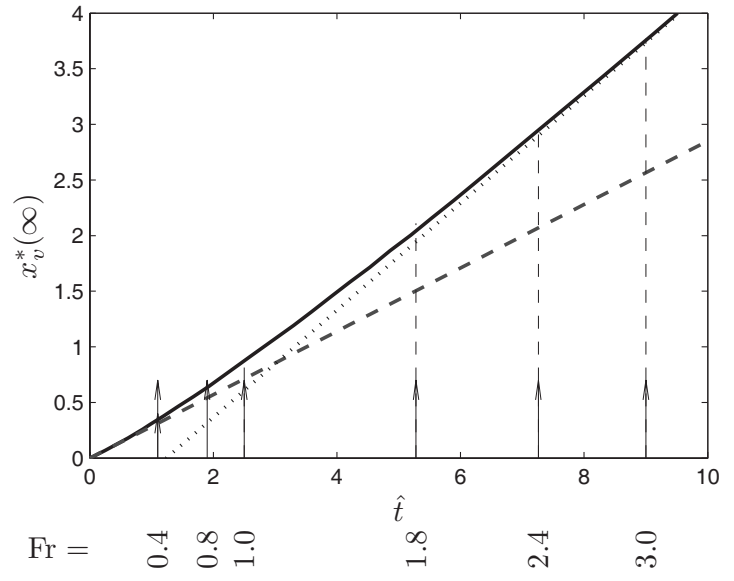

FIG. 14. Axial location of the leading vortex ring of a starting, homogeneous jet, $\mathrm{Fr}=\infty$. The solid line represents the location of the vortex ring, whereas the dashed line represents the initial velocity, $V_{0}$, and the dotted line the final one, $V_{\infty}=0.5$. The vertical lines are plotted when the vortices from jets with different Froude numbers reach their maximum penetration depth.

When the negative velocity induced by gravity effects is comparable to the velocity of the leading vortex ring of the homogeneous jet $(\mathrm{Fr}=\infty)$, the penetration depth nearly reaches its maximum value. This occurs at times $\hat{t} \approx 2.5 \mathrm{Fr}$ (see Fig. 13) and it explains also the collapse of the $x_{v}^{*} / \mathrm{Fr}-\hat{t} / \mathrm{Fr}$ curves observed for $\mathrm{Fr} \leq 1$, displayed in Fig. 13. Indeed, the examination of the time evolution of the vortex ring position for the homogeneous case, shown in Fig. 14 , reveals that it starts moving at a nearly constant speed until $\hat{t} \approx 2$. Afterward it exhibits an acceleration stage, until it reaches its final speed, at approximately $\hat{t} \approx 6\left(V_{\infty} \approx 0.5\right.$ according to the slug-flow model $\left.{ }^{3}\right)$. Therefore, for starting jets such that the leading vortex ring reaches its maximum penetration before this acceleration stage occurs, the assumption of constant velocity, $x_{v(\infty)}^{*}=V_{0} t^{*}$, describes fairly well the observed $x_{v}^{*} / \mathrm{Fr}-\hat{t} / \mathrm{Fr}$ trajectories. Notice that this condition is equivalent to $\mathrm{Fr} \lesssim 1$. On the contrary, those vortices that still have not been significantly affected by gravity at $\hat{t} \approx 2$, feel the acceleration stage and thus do not satisfy the condition of constant initial velocity $V_{0}$. For these cases Eq. (13) is no longer valid and the $x_{v}^{*} / \mathrm{Fr}-\hat{t} / \mathrm{Fr}$ curves depend on the Froude number.

To clarify this point, vertical (i.e., constant time) lines have been plotted in Fig. 14 indicating the time at which the vortices corresponding to different Froude numbers reach their maximum penetration depth. Notice that vertical lines, corresponding to subcritical cases, are placed at times when the velocity of the homogeneous vortex ring has not changed significantly with respect to its initial value.

A possible explanation for the acceleration stage of the vortex ring for $\mathrm{Fr}=\infty$ is that at the beginning of the stage, the vortex has engulfed enough vorticity to exhibit a selfinduced velocity faster than the initial convective one, $V_{0}$. Therefore, vortex rings of different Froude numbers show coincident values of their axial locations during these first instants, however, subcritical vortices never reach this selfinduced velocity because gravity forces push them away before this can occur. 
To conclude this section, a final comment must be made about the apparent universality of the vortex gravitational acceleration factor, $\alpha$, used in Eq. (12), that leads to the collapse of the different curves shown in Fig. 12. It has been shown in numerical simulations that the average density of the leading vortex ring is not $\rho_{j}$ but a value between $\rho_{j}$ and $\rho_{0}$ due to the entanglement of jet and ambient fluid patches in the vortex core and to molecular mixing. Therefore, it should be more appropriate to say that the actual acceleration acting on the vortex is $\alpha g^{*}$ rather than $g^{*}$, where $\alpha<1$. The value obtained from Fig. 12 is relatively low, about $\alpha \approx 0.1$, indicating that the vortex ring entrains more ambient fluid than it does from the jet. Furthermore, the fact that this parameter is nearly constant for all Froude numbers, density ratios and Reynolds numbers explored in this work indicate that the proportions in which the jet and ambient fluids are mixed are almost unaffected by these parameters. It is important to remark that this parameter $\alpha$ must not be understood as the actual mixing ratio existing inside the vortex ring but as a free parameter obtained by fitting the kinematic model described by Eq. (12) to the numerical and experimental data. The parameter $\alpha$ would be exactly the mixing ratio only if the vortex ring was a nondeformable object of constant size, which obviously is not the case during its formation process, when the vortex grows and changes its shape as a result of the vorticity redistribution occurring through this stage. Therefore, the value $\alpha=0.1$ does not mean that the final composition of the vortex ring is $10 \%$ jet fluid and $90 \%$ ambient fluid.

\section{B. Evolution of the total circulation}

The assumption that the vortex location can be expressed as the location of the starting jet at $\mathrm{Fr}=\infty$, plus a term that accounts for the effect of the Froude number can also be extended to describe the evolution of the circulation reported in Sec. V for finite Froude numbers. The vorticity equation in an axisymmetric, high Reynolds number, laminar flow with nonuniform density is given by ${ }^{12}$

$$
\frac{\partial}{\partial t}\left(\frac{\omega}{r}\right)+\nabla \cdot\left(\vec{u} \frac{\omega}{r}\right)=\frac{(\nabla \rho \wedge \nabla p)_{\theta}}{r \rho^{2}} .
$$

Equation (14) can be integrated over the total fluid volume, $V$, and divided by $2 \pi$ to yield

$$
\begin{gathered}
\frac{\mathrm{d}}{\mathrm{d} t} \int_{S_{0}} \omega \mathrm{d} r \mathrm{~d} x+\frac{1}{2 \pi} \int_{V} \nabla \cdot\left(\vec{u} \frac{\omega}{r}\right) \mathrm{d} V \\
=\int_{S_{0}} \frac{(\nabla \rho \wedge \nabla p)_{\theta}}{\rho^{2}} \mathrm{~d} r \mathrm{~d} x,
\end{gathered}
$$

where in the first and third terms, the differential volume has been substituted by $\mathrm{d} V=2 \pi r \mathrm{~d} r \mathrm{~d} x$, transforming the volume integral into a surface integral over a constant- $\theta$ surface, $S_{0}$ (see Fig. 15). The first integral can now be rewritten as

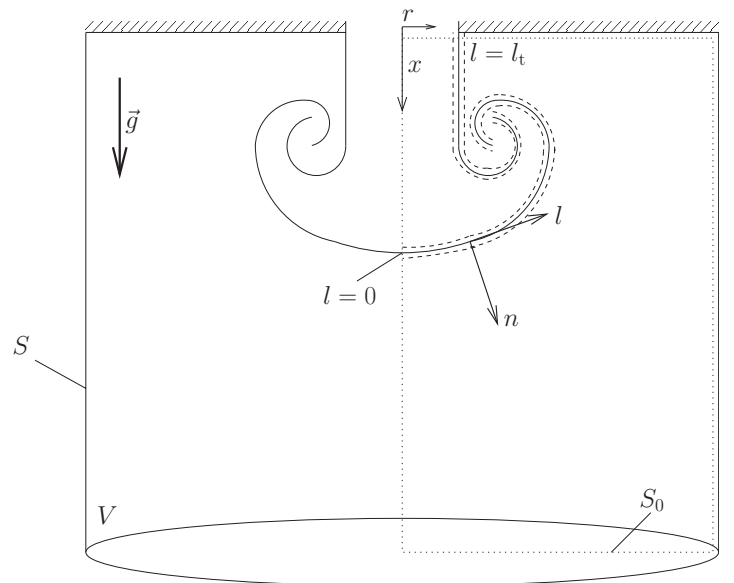

FIG. 15. Sketch of the coordinate system employed to evaluate the baroclinic production integral.

$$
\frac{\mathrm{d}}{\mathrm{d} t} \int_{S_{0}} \omega \mathrm{d} r \mathrm{~d} x=\frac{\mathrm{d} \Gamma}{\mathrm{d} t} .
$$

On the other hand, application of Gauss' theorem allows us to write the second integral in Eq. (15) in the following way:

$$
\int_{V} \nabla \cdot\left(\vec{u} \frac{\omega}{r}\right) \mathrm{d} V=\oint_{S} \frac{\omega}{r} \vec{u} \cdot \vec{n} \mathrm{~d} S,
$$

which represents the net vorticity flux across the control surface $S$. Furthermore, the only vorticity flux across the integration surface is that occurring through the injection orifice, and does not depend either on the Froude number or on the density ratio. Applying these arguments to the vorticity equation in the case $\mathrm{Fr}=\infty$, one obtains

$$
\frac{1}{2 \pi} \int_{V} \nabla \cdot\left(\vec{u} \frac{\omega}{r}\right) \mathrm{d} V=-\left.\frac{\mathrm{d} \Gamma}{\mathrm{d} t}\right|_{\mathrm{Fr}=\infty} .
$$

Finally, the third integral in Eq. (15) represents the baroclinic production of vorticity and will be denoted hereafter by $\mathrm{d} \Gamma /\left.\mathrm{d} t\right|_{\text {bar }}$. Thus, it can be concluded that the difference between the circulation of neutrally buoyant jets and that of nonhomogeneous jets is due to the generation of baroclinic vorticity,

$$
\frac{\mathrm{d} \Gamma}{\mathrm{d} t}=\left.\frac{\mathrm{d} \Gamma}{\mathrm{d} t}\right|_{\mathrm{Fr}=\infty}+\left.\frac{\mathrm{d} \Gamma}{\mathrm{d} t}\right|_{\mathrm{bar}} .
$$

In order to estimate the baroclinic production occurring for $\mathrm{Fr}<\infty$, the same reasoning described by Iglesias et al. ${ }^{12}$ can be followed. First of all, since in our experiments and simulations the Reynolds and Schmidt numbers are large, it is assumed that the density gradients, and thus baroclinic production, is confined to the jet-ambient interface, and the corresponding surface integral in Eq. (15) must be only evaluated along a thin layer surrounding the interface (see Fig. 15). This integral can be easily calculated using a new system of coordinates $(n, l)$, where $n$ is the normal to the density interface and $l$ is the coordinate tangent to this inter- 


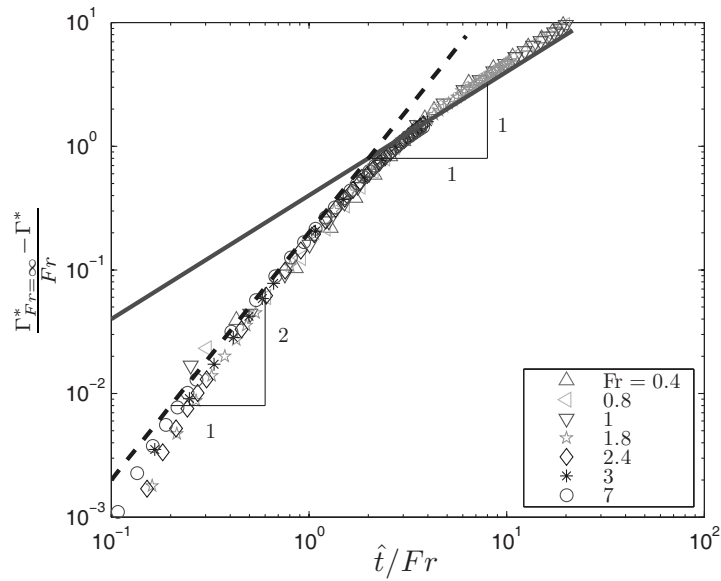

FIG. 16. Contribution of the baroclinic production to the total circulation. The dashed line is the prediction of the model for short times $\left(\sim \hat{t}^{2}\right)$ whereas the thick solid line goes as $\sim \hat{t}$ (the numerical simulations correspond to the same cases as in Fig. 12).

face. In this new coordinate system, and according to the hypothesis that density variations occur only at $n=0$, the baroclinic integral can be expressed as

$$
\begin{aligned}
\left.\frac{\mathrm{d} \Gamma}{\mathrm{d} t}\right|_{\text {bar }} & =\int_{S_{0}} \frac{(\nabla \rho \wedge \nabla p)_{\theta}}{\rho^{2}} \mathrm{~d} r \mathrm{~d} x \\
& =\int_{0_{-}}^{0_{+}} \frac{1}{\rho^{2}} \frac{\partial \rho}{\partial n} \mathrm{~d} n \int_{0}^{l_{t}} \frac{\partial p}{\partial l} \mathrm{~d} l \\
& =-\left[p\left(x_{f}, 0\right)-p(0, D / 2)\right]\left(\frac{1}{\rho_{j}}-\frac{1}{\rho_{0}}\right),
\end{aligned}
$$

where $\rho_{0}$ is the density of the outer fluid and $x_{f}$ represents the axial position of the jet front, $l=0$. Here the pressure at $x=0$ can be set to zero without loss of generality and the pressure at $x=x_{f}$ can be assumed to be $p\left(x_{f}, 0\right) \approx \rho_{0} g x_{f}$. Indeed, the pressure at this point is expected to differ from that of a point located at the same $x=x_{f}$, but far away from the axis, $r \rightarrow \infty$, in an amount of the order $\rho_{j} U_{j}^{2} \ll \rho_{0} g x_{f}$, as shown by

$$
\frac{\rho_{j} U_{j}^{2}}{\rho_{0} g x_{f}}=\frac{\rho_{j}}{\rho_{0}} \frac{U_{j}^{2}}{g D} \frac{D}{x_{f}}=\operatorname{Fr} \frac{\Delta \rho}{\rho_{0}} \frac{D}{x_{f}} .
$$

It should be kept in mind that the parameter $\Delta \rho / \rho_{0}$ is typically small in all the cases investigated here, specially for Fr $>1$. Using the above assumption Eq. (20) reduces to

$$
\left.\frac{\mathrm{d} \Gamma}{\mathrm{d} t}\right|_{\mathrm{bar}}=-\rho_{0} g x_{f}\left(\frac{1}{\rho_{j}}-\frac{1}{\rho_{0}}\right)=-\frac{1}{\mathrm{Fr}} x_{f}^{*} U_{j}^{2} .
$$

Since initially the front position, $x_{f}$, does not depend on the Froude number and advances at a nearly constant speed proportional to $U_{j}, x_{f}^{*} \sim t^{*}$, one obtains

$$
\Gamma_{\mathrm{bar}}^{*} \sim-\frac{t^{* 2}}{\mathrm{Fr}} \text {. }
$$

Figure 16 shows the dimensionless circulation difference, $\left(\Gamma_{\mathrm{Fr}=\infty}^{*}-\Gamma^{*}\right) / \mathrm{Fr}$ as a function of $\hat{t} / \mathrm{Fr}$ in a logarithmic plot. It can be observed that for short times it follows the trend $\left(\Gamma_{\mathrm{Fr}=\infty}^{*}-\Gamma^{*}\right) / \mathrm{Fr} \sim(\hat{t} / \mathrm{Fr})^{2}$ predicted by the model, provided in Eq. (23). For larger times, $\hat{t} / \mathrm{Fr} \gtrsim 2.5$, the baroclinic production slows down and starts increasing linearly with time (see Fig. 16). Examination of Fig. 13 reveals that this change in slope coincides with the time when the leading vortex location, and therefore the jet's front, reaches a maximum penetration, thus $\mathrm{d} \Gamma /\left.\mathrm{d} t\right|_{\text {bar }}$ becomes constant in agreement with the proposed model.

\section{CONCLUSIONS}

Negatively buoyant starting jets have been studied experimentally and numerically in order to clarify the role of buoyancy forces in their development. When the Reynolds number is large enough and the density and viscosity ratios are close to unity, the densimetric Froude number, Fr, emerges as the only relevant parameter of the flow. The evolution of a number of important jet features such as the trajectory of the leading vortex ring, formation number, final penetration depth, as well as the vorticity fields and total circulation have been analyzed for starting jets with different Froude numbers and compared to the case of neutrally buoyant jets.

A first conclusion of the study is the existence of two different flow regimes: For Froude numbers larger than about $\mathrm{Fr}_{c} \sim 1$, the leading vortex ring detaches from the injection orifice and advances downstream into the stagnant ambient followed by a trailing jet, eventually reaching a maximum penetration depth and loosing the axial symmetry for finite Froude numbers. On the other hand, for $\mathrm{Fr}<1$, the leading vortex ring is pushed upstream by buoyancy forces and spreads out radially before it has time to develop a selfinduced velocity. Analysis of the leading vortex location history reveals that the transition between these two regimes occurs when the gravitational time, the time that gravity takes to induce a velocity equal to the injection one, is of the order of the vortex ring formation time, $D / U_{j}$. This condition is equivalent to $\mathrm{Fr} \sim 1$. Based on these ideas, a simple model has been proposed to describe the location of the buoyant leading vortex ring as a function of time. The key idea is to decompose the vortex velocity, and thus its position, as the sum of that of the homogenous vortex ring minus a term that accounts for the effect of gravitational acceleration. The model, which only has one free parameter, describes fairly well the trends observed in both simulations and experiments.

Regarding the vorticity fields, the main difference observed when they are compared to the vorticity distribution observed for neutrally buoyant jets is the existence of a layer of opposite sign to the one injected that moves upward due to buoyancy effects. Examination of the vorticity equation reveals that the origin of this negative vorticity layer is the baroclinic production. Furthermore, a model is also proposed to evaluate the baroclinic production and therefore to explain the observed evolution of the total circulation found in the flow.

Finally, the effect of the Froude number on the vortex ring formation number, $F$, has been evaluated. For $\mathrm{Fr}>1$, when this parameter can be defined, the formation number 
increases with the Froude number, eventually reaching the range of values reported in literature for the homogeneous case, $F \sim 3-4$. This is consistent with the smaller size of the negatively buoyant vortex rings compared to their neutrally buoyant counterparts. Indeed, as described above, part of the fluid transported by the trailing jet is taken from the vortex ring and moved upward by buoyancy forces during the vortex ring formation process.

\section{ACKNOWLEDGMENTS}

This work was supported by the Spanish Ministry of Education under Project Nos. DPI2008-06624-C03-02 and ENE2008-0615-C04. This work has been extracted from the Ph.D. thesis of Marugán-Cruz. ${ }^{26}$

${ }^{1}$ M. Gharib, E. Rambod, and K. Shariff, "A universal time scale for vortex ring formation," J. Fluid Mech. 360, 121 (1998).

${ }^{2}$ D. I. Pullin, "Vortex ring formation at tube and orifice openings," Phys. Fluids 22, 401 (1979).

${ }^{3}$ K. Shariff and A. Leonard, "Vortex rings," Annu. Rev. Fluid Mech. 24, 235 (1992).

${ }^{4}$ N. Didden, "Formation of vortex rings: Rolling-up and production of circulation," Z. Angew. Math. Phys. 30, 101 (1979).

${ }^{5}$ W. Zhao, S. H. Frankel, and L. Mongeau, "Effects of trailing jet instability on vortex ring formation," Phys. Fluids 12, 589 (2000).

${ }^{6}$ G. Pawlak, C. Marugán-Cruz, C. Martínez-Bazán, and P. García-Hrdy, "Experimental characterization of starting jets dynamics," Fluid Dyn. Res. 39, 711 (2007).

${ }^{7}$ L. Pantzlaff and R. M. Lueptow, "Transient positively and negatively buoyant turbulent rounds jets," Exp. Fluids 27, 117 (1999).

${ }^{8}$ T. S. Turner, "Jets and plumes with negative or reversing buoyancy," J. Fluid Mech. 26, 779 (1966)

${ }^{9}$ P. Philippe, C. Raufaste, P. Kurowski, and P. Petitjeans, "Penetration of a negatively buoyant jet in a miscible liquid," Phys. Fluids 17, 053601 (2005).

${ }^{10}$ W. Lin and S. W. Armfield, "Direct simulation of weak axisymmetric fountains in a homogeneous fluid," J. Fluid Mech. 403, 67 (2000).

${ }^{11}$ R. W. Cresswell and R. T. Szczepura, "Experimental investigation into a turbulent jet with negative buoyancy," Phys. Fluids A 5, 2865 (1993).

${ }^{12}$ I. Iglesias, M. Vera, A. L. Sánchez, and A. Liñán, "Simulations of starting gas jets at low Mach numbers," Phys. Fluids 17, 038105 (2005).

${ }^{13}$ R. P. Satti and A. K. Agrawal, "Computational study of buoyancy effects in a laminar starting jet," Int. J. Heat Fluid Flow 29, 527 (2008).

${ }^{14}$ M. Rosenfeld, E. Rambod, and M. Gharib, "Circulation and formation of laminar vortex rings," J. Fluid Mech. 376, 297 (1998).

${ }^{15}$ R. C. Weast, CRC Handbook of Chemistry and Physics (CRC, Cleveland, 1985).

${ }^{16}$ R. H. Perry and D. W. Green, Perry's Chemical Engineers' Handbook (McGraw-Hill, New York, 2007).

${ }^{17}$ N. B. Kaye and G. R. Hunt, "Weak fountains," J. Fluid Mech. 558, 319 (2006).

${ }^{18}$ P. D. Friedman and J. Katz, "The flow and mixing mechanisms caused by the impingement of an immiscible interface with a vertical jet," Phys. Fluids 11, 2598 (1999).

${ }^{19} \mathrm{H}$. Zhang and R. Baddour, "Maximum penetration of vertical round dense jets at small and large Froude numbers," J. Hydraul. Eng. 124, 550 (1998).

${ }^{20}$ W. D. Baines, J. S. Turner, and I. H. Campbell, "Turbulent fountains in an open chamber," J. Fluid Mech. 212, 557 (1990).

${ }^{21}$ P. N. Papanicolaou and T. J. Kokkalis, "Vertical buoyancy preserving and non-preserving fountains, in a homogeneous calm ambient," Int. J. Heat Mass Transfer 51, 4109 (2008).

${ }^{22}$ J. Westerweel, Digital Particle Velocimetry. Theory and Application (Delft University Press, Delft, 1993).

${ }^{23}$ M. Raffel, C. Willert, and J. Kompenhans, Particle Image Velocimetry-A Practical Guide (Springer-Verlag, Berlin, 1998).

${ }^{24}$ A. Acosta-Iborra, "Métodos autoadaptativos para la derivación de campos de velocidad procedentes de la técnica de velocimetría por imagen de partículas (PIV)," Ph.D. thesis, Universidad Carlos III de Madrid, 2004.

${ }^{25}$ J. E. Carter, J. Soria, and T. T. Lim, "The interaction of the piston vortex with a piston-generated vortex ring," J. Fluid Mech. 499, 327 (2004).

${ }^{26}$ C. Marugán-Cruz, "Experimental and numerical analyses of starting jets with different jet-to-ambient density ratios," Ph.D. thesis, Universidad Carlos III de Madrid, 2008. 
Physics of Fluids is copyrighted by the American Institute of Physics (AIP). Redistribution of journal material is subject to the AIP online journal license and/or AIP copyright. For more information, see http://ojps.aip.org/phf/phfcr.jsp 